\title{
Resistant Erythema Nodosum-Remembering an Old Treatment (Potassium Iodide)
}

Heather M Lake, Elaine P Alexander, Zhanna Mikulik ${ }^{1}$ and Kevin V Hackshaw

The Ohio State University, Division of Immunology/Rheumatology, William Davis Medical Research Center, 480 Medical Center Drive, Columbus, Ohio, 43210-1228, USA

"Corresponding Author: Kevin V Hackshaw, The Ohio State University Wexner Medical Center, Division of Immunology/Rheumatology, William Davis Medical Research Center, 480 Medical Center Dr, Columbus, OH 43210-1228, USA, Tel: (614) 293-8093; E-mail: Kevin.Hackshaw@osumc.edu

Received date: December 14, 2016; Accepted date: February 25, 2017; Published date: February 28, 2017

Copyright: (C 2017 Lake HM. This is an open-access article distributed under the terms of the Creative Commons Attribution License, which permits unrestricted use, distribution, and reproduction in any medium, provided the original author and source are credited.

\section{Abstract}

This is a case report of a patient with resistant erythema nodosum. Despite multiple traditional treatments, the patient did not improve until the use of potassium iodide, an under used treatment for EN. Also included is a review of EN including clinical features, histology, and treatment. Potassium iodide as a treatment for treatment resistant EN is discussed in detail including possible mechanism of action, dosage, adverse effects, and contraindications.

Keywords: Nodules; Potassium iodide; Erythema nodosum

Abbreviations EN: Erythema Nodosum; KI: Potassium Iodide

\section{Case Report}

A 55 year-old Caucasian female with a past medical history of refractory hepatitis C, coronary artery disease, COPD, diabetes, hypertension, obesity, and GERD who presented to our Rheumatology clinic in January complaining of lesions on her right leg.

Her lesions started nine months prior as a small spot over her right calf which subsequently grew into nodules. They were tender to touch and red in appearance. She was treated by her primary care physician for cellulitis with several courses of antibiotics including doxycyline without improvement. She was then treated with steroids with no change. She was started on colchicine, which did not help.

Her outside labs were reviewed, which included a rheumatoid factor, sedimentation rate, serum protein electrophoresis, complete blood count, creatinine, ANCA, compliment levels including (CH50, C3, C4), C-reactive protein, lyme test, and ANA which were all negative or within normal limits. Her liver enzymes were elevated as well as her angiotensin converting enzyme at 93 with normal being less than 68. An X-ray of her chest was unremarkable. She denied signs or symptoms consistent with connective tissue disease or systemic vasculitis. She denied fevers, weight loss or joint pain. On this initial visit, her physical exam was unremarkable with the exception of several nodules located on her shins, which were tender to palpation, erythematous and ranged in size from 1-5 cm. A clinical diagnosis of erythema nodosum was made and she was started on Dapsone. Hydroxychloroquine was considered but there was concern for liver toxicity with Hepatitis-C.

Initially, her nodules decreased in size on Dapsone, but she then developed new nodules on her ankles. She continued to have normal inflammatory markers and elevated liver enzymes on repeat labs. Dapsone was discontinued secondary to the development of lesions while on this medication. Prednisone was started at a low dose of $5 \mathrm{mg}$ daily. She tolerated low dose prednisone but did not have improvement. Her dose was increased to $10 \mathrm{mg}$ daily, but she continued to develop new lesions.

She was referred to dermatology who tried high dose steroids beginning at $60 \mathrm{mg}$ with taper over the next two weeks but the nodules did not respond. She was started on a non-steroidal anti-inflammatory, Mobic $7.5 \mathrm{mg}$ daily, at our clinic without improvement. Dermatology then arranged for a biopsy. Biopsy was completed in September 2008 from a large extensor surface nodule of $5 \mathrm{~cm}$ on the thigh and erythema nodosum was confirmed.

At follow up she was started on potassium iodide $65 \mathrm{mg} / \mathrm{ml}$ with a total dose of $133 \mathrm{mg}$ three times daily with half a glass of water. Soon after starting potassium iodide, she had significant improvement in her nodules. All nodules disappeared after one month of treatment with the exception of a small nodule on her left great toe. She denied metallic taste, soreness of her teeth and gums, watery mouth, or burning of her mouth. She had slight diarrhea and nausea which she tolerated well. A taper of potassium iodide was planned, but instead, it was stopped secondary to an elevation in her TSH. This returned to normal with repeat labs completed three weeks after discontinuation of the medication. Despite discontinuation she has not had recurrence of the erythema nodosum.

\section{Discussion}

Erythema Nodosum (EN) is characterized by red or violet subcutaneous nodules that usually develop over the extensor surfaces of the lower extremities. EN is presumed to represent a delayed hypersensitivity reaction to a wide variety of inciting factors. It may be triggered by a variety of stimuli including various infectious agents, drugs, diseases (eg, Sarcoidosis, IBD, and other autoimmune disorders), pregnancy and malignancy [1]. EN can occur at any age but most cases appear between the second and fourth decades of life. EN occurs 3-6 times more frequently in women than men. The majority of patients are found to have had a recent streptococcal infection or no identifiable cause, and in latter cases, symptomatic relief constitutes the only treatment.

The painful, nodules that develop on the anterior surface of the legs evolve into bruise-like lesions that resolve without scarring over a two 
to eight week period. Nodules may appear anywhere there is fat under the skin, including, the thighs, arms, trunk, face and neck but absence of nodules on the legs is atypical. The nodules are $1-5 \mathrm{~cm}$ in diameter, and individual nodules may coalesce to form large areas of hardened skin. At first, the nodules are bright red in color and are raised slightly above the skin. Within a few days, they become flat, with a livid red or purplish color. Finally, they exhibit a yellow or greenish appearance often taking on the look of a deep bruise. Polyarthralgias, fever and malaise frequently accompany the rash. The erythrocyte sedimentation rate may be elevated [2].

Diagnosis is usually clinical but a deep punch biopsy or an incisional biopsy may be performed in cases where the diagnosis is unclear. Recurrences are not uncommon. Etiologic workup should include complete blood count, sedimentation rate, ASO titer, urinalysis, throat culture, intradermal tuberculin test and chest X-ray [1].

Histologically, the rash is a panniculitis involving inflammation of the septa in the subcutaneous fat tissue, usually without associated vasculitis. EN is the most common form of panniculitis (inflammation of the subcutaneous fat). The composition of the inflammatory infiltrate in the septa varies with the age of the lesion. In the early lesion edema, hemorrhage and neutrophils are responsible for the septal thickening, whereas fibrosis, periseptal granulation tissue, lymphocytes, and multinucleated giant cells are the main finding in late stage lesions. A histopathologic hallmark of erythema nodosum is the presence of Miescher's radial granulomas, which consist of small, well defined nodular aggregations of small histiocytes arranged radially around a central cleft of variable shape [2]. This was initially described by Miescher. Although Miescher's granulomata are a useful indicator of EN the typical radial granulomas are present in less than $75 \%$ of cases. As suggested by Miescher, they are seen in very early disease as early as day 2 therefore biopsy timing is crucial for their detection [3].

EN is usually self-limited or resolves with treatment of the underlying disorder if identified. Relief of the discomfort associated with the rash itself or the accompanying arthralgias can be accomplished with a variety of treatments, including bed rest, leg elevation, compressive bandages, wet dressings, nonsteroidal anti- inflammatory drugs, aspirin, colchicine and hydroxychloroquine. Glucocorticoids are usually not necessary for idiopathic EN. A list of treatment options with their corresponding level of evidence is listed in (Table 1).

\begin{tabular}{|l|l|}
\hline NSAIDs & Case reports \\
\hline Colchicine & Case series \\
\hline Potassium lodide & Case series \\
\hline Heparin via occlusion (topical) & Case reports \\
\hline Prednisone & Case reports \\
\hline Intra-lesional corticosteroids & Case reports \\
\hline Dapsone & Case reports \\
\hline Hydroxychloroquine & Case reports \\
\hline Anti-TNF monoclonal antibodies & Case reports \\
\hline Thalidomide & Case reports \\
\hline Methotrexate & Case reports \\
\hline Azathioprine & Case reports \\
\hline Rituximab & Case reports \\
\hline
\end{tabular}

Table 1: Level of Evidence for Erythema Nodosum Treatments

Potassium iodide (360 to $900 \mathrm{mg}$ daily in three divided doses) can be used. This treatment was "re-discovered" after reports of treatment successes with other forms of panniculitis that had in turn been suggested by the common use of iodide by turn of the century dermatologists. The basis for the documented benefit of potassium iodide in treating EN is unclear. The evidence for efficacy of pharmacologic treatment for EN is based almost exclusively on small observational studies (Figure 1), making treatment recommendations difficult. Choices of agent, dose, and duration thus remain matters of style and preference for the individual clinician [2].
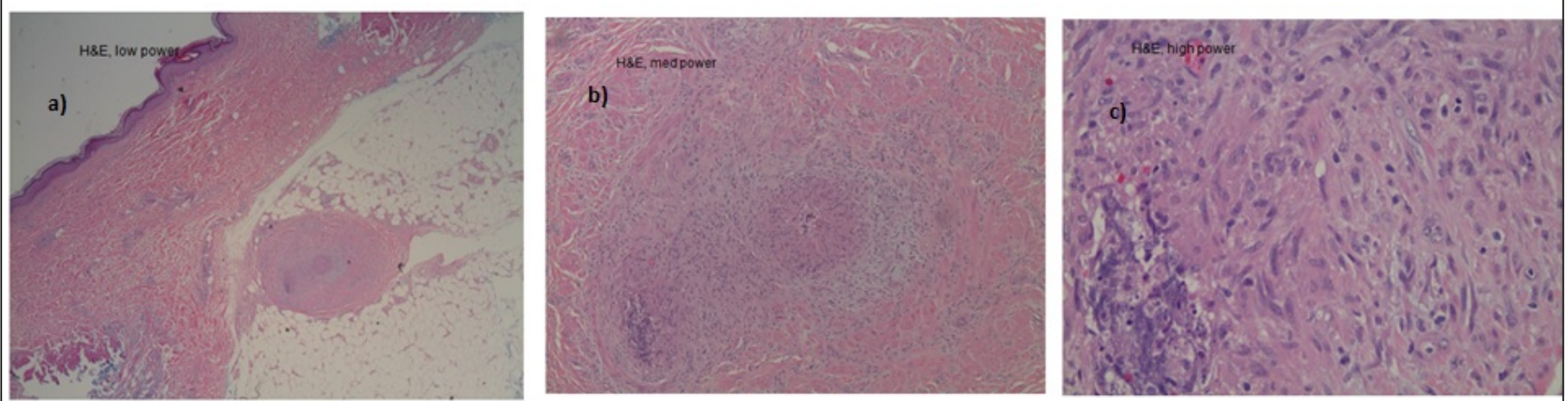

Figure 1: Low, medium and high magnification by hematoxylin and eosin showing evidence for a septal panniculitis with mixed cellular infiltrate of lymphocytes, histiocytes, giant cells and occasional eosinophils with absence of vasculitis.

Potassium iodide (KI) was first discovered in seaweed in the early $1800 \mathrm{~s}$ and was initially used to treat thyroid disease. Today, dermatologists can use this to treat a variety of dermatologic conditions. KI is a compound made of $76 \%$ of the halogen iodine and
$23 \%$ of the alkali metal potassium. The KI dose used to treat dermatoses is much higher than those used to treat thyrotoxicosis or protect against radiation. Typical oral dose is $300 \mathrm{mg}$ (approximately 6 drops of saturated solution SSKI) 3 times daily. After ingestion KI is 
readily absorbed in the intestinal tract and distributes rapidly to the extracellular space [4]. The mechanism of action of KI in EN is largely unknown, but it is thought that it causes heparin release from mast cells and heparin acts to suppress delayed hypersensitivity reactions. There is also evidence that it inhibits neutrophil chemotaxis $[5,6]$ as well as evidence that it blocks the neutrophil's ability to generate toxic free radicals [6]. Purported mechanism of actions are depicted in (Figure 2). KI has also been used in a variety of granulomatous diseases including granulomatosis with polyangiitis (GPA) suggesting an antigranulomatous effect, however more research into exact pathogenesis is required [6].

\section{KI $\rightarrow$ Neutrophil Chemotaxis $\downarrow$}

$\downarrow$ Free Radicals

Mast Cell $\rightarrow$ Heparin Delayed Hypersensitivity

Figure 2: Potassium iodide purportedly stimulates mast cells to release heparin which may lead to suppression of delayed hypersensitivity reactions. Potassium iodide also may decrease free radical formation and neutrophil chemotaxis.

When patients are given $\mathrm{KI}$, they should be advised regarding possible adverse reactions including irregular heartbeat, confusion, tiredness, fever, skin rash, goiter, salivary gland swelling/tenderness, thyroid adenoma, swelling of neck/throat, myxedema, lymph node swelling, hyper/hypothyroidism, diarrhea, gastrointestinal bleeding, metallic taste, nausea, stomach pain, vomiting, numbness/tingling, weakness, joint pain, and hypersensitivity reactions. Contraindications to this medication include Hypersensitivity to iodine or any component of the formulation, hyperkalemia, pulmonary edema, impaired renal function, hyperthyroidism, iodine-induced goiter, dermatitis herpetiformis, hypocomplementemic vasculitis and pregnancy as it can produce a goiter in the fetus. You must use it with caution in patients taking medications that increase potassium (eg, ACEI, potassium-sparing diuretics, potassium containing salt substitutes). When $\mathrm{KI}$ is used for more than one month a screening TSH is prudent to ensure that iodide-induced hypothyroidism does not ensue. If this is detected, discontinuing KI will usually result in normal T4, T3, and TSH with one month [4].

In our patient, the cause of EN was felt to be idiopathic, but EN has been reported to be associated with HCV infection, usually related to underlying cryoglobulinemia or vasculitis $[7,8]$, both of which were negative in our patient. However a causal relationship cannot be excluded. With new treatments for HCV on the rise, it would have been interesting to see if the EN resolved with definitive treatment of HCV. While treatment of the underlying condition if known or an anti-inflammatory for symptomatic relief may be all that is necessary in most cases of idiopathic EN, it is important to remember KI for those individuals who do not have contraindications.

\section{References}

1. Gonzalez-Gay MA, Garcia-Porrua C, Pujol RM (2001) Erythema nodosum: a clinical approach. Clin Exp Rheumatol 19:365.

2. Requena L, MD Yus ES (2007) Erythema nodosum. Seminars in Cutaneous Medicine and Surgery 26: 114-125.

3. White WL, Wieselthier JS, Hitchcock MG (1996) Panniculitis: recent developments and observations. Seminars in Cutaneous Medicine and Surgery 15: 278-299.

4. Sterling JB, Heymann WR (2000) Potassium iodide in dermatology: A 19th Century Drug for the 21st Century-Uses, Pharmacology, Adverse effects, and Contraindications. The American Academy of Dermatology 43:691-697.

5. Horio T, MD Imamura S, Danno K (1981) Potassium Iodide in the treatment of erythema nodosum and nodular vasculitis. Archives Dermatology 117: 29-31.

6. Gilchrist H, Patterson JW (2010) Erythema nodosum and erythema induratum (nodular vasculitis): diagnosis and management. Dermatologic Therapy 23: 320-327.

7. Hadziyannis SJ (1998) Skin diseases associated with hepatitis C virus infection. Journal of the European Academy of Dermatology and Venereology 10: 12-21.

8. Blake T, Manahan M, Rodins K (2014) Erythema Nodosum - A Review of an Uncommon Panniculitis. Dermatology Online Journal 20:4. 